\title{
LA EXTENSION DEL GLACIARISMO CUATERNARIO EN EL PARQUE NACIONAL DE ORDESA Y MONTE PERDIDO
}

\author{
Carlos MARTI BONO y José Ma GARCIA RUIZ \\ Instituto Pirenaico de Ecología \\ Campus de Aula Dei Zaragoza
}

\begin{abstract}
Resumen: Se revisa la extensión máxima alcanzada por los glaciares del Parque Nacional de Ordesa y Monte Perdido durante el Pleistoceno reciente. La gran altitud del macizo de Monte Perdido permitió la existencia de potentes lenguas de hielo que tenían su zona terminal en torno a $900 \mathrm{~m}$, excepto el glaciar de Escuaín y el de Pardina-Vió, que en razón de la menor altitud y extensión de sus respectivas cabeceras finalizaban muy por encima de ese límite. La ausencia de morrenas frontales obliga a reconstruir tal extensión por medio de morrenas laterales y otros depósitos (terrazas fluvioglaciares, brechas, conos de deyección). Se alude igualmente a otros depósitos glaciares correspondientes a fases de retracción, pudiendo distinguirse una fase de glaciares de altitud, otra de glaciares de circo y la Pequeña Edad del Hielo.
\end{abstract}

Palabras clave: Glaciares cuaternarios, morrenas terminales, morrenas laterales, Parque Nacional de Ordesa, Pirineos.

\begin{abstract}
The maximal extent reached by the glaciers in the Ordesa and Monte Perdido National Park during the young Pleistocene is discussed. The great height of the Monte Perdido massif allowed the existence of deep ice tongues that had their final area around $900 \mathrm{~m}$ a.s.l., except for the Escuain and Pardina-Vió glaciers, that owing to the lower altitude and extent of their respective headwaters finished higher. The absence of frontal moraines obligates us to reconstruct such extent by means of lateral moraines and other deposits (flluvioglacial terraces, screes, alluvial fans). Likewise, other glacial deposits are cited, belonging to retreat phases. A phase of altitude glaciers, other of cirque glaciers and the Little Ice Age can be distinguished.
\end{abstract}

Key words: Quaternary glaciers, final moraines, lateral moraines, Ordesa National Park, Pyrenees. 


\section{INTRODUCCION}

Junto con la estructura, la acción de los glaciares ha contribuido decisivamente a explicar algunos de los rasgos más importantes del relieve del Parque Nacional de Ordesa y Monte Perdido. Cualquiera que sea la escala desde la que se contempla este territorio, se encuentran herencias procedentes de la erosión ejercida por el paso del hielo sobre rocas de diferente resistencia; de igual manera, son numerosos los ejemplos de depósitos abandonados por los glaciares en diferentes momentos del Pleistoceno Superior y del Holoceno.

La notable elevación del relieve -cuyas divisorias más altas superan frecuentemente los $3.000 \mathrm{~m}$ - y su masividad explican la existencia de numerosas evidencias glaciares, tanto en las proximidades de las líneas de cumbres como a lo largo de los valles principales. El objetivo de este trabajo es reconstruir, a partir de la distribucción espacial de las formas y los sedimentos glaciares, la extensión alcanzada por las grandes masas de hielo en el territorio del Parque Nacional y áreas próximas durante el Cuaternario reciente. Conviene tener presente que se trata de un problema todavía no resuelto, pues son muy escasas las referencias bibliográficas sobre el glaciarismo del Parque Nacional, la mayoría bastante antiguas y referidas a aspectos muy parciales (ver Penk, 1883; Hernández Pacheco, 1920; Obermaier, 1921; Gómez de Llarena, 1936; Hernández Pachecho y Vidal Box, 1946; Boyé, 1952; NicolásMartínez, 1981). Recientemente García Ruiz y Martí Bono (1993) han elaborado el Mapa Geomorfológico del Parque Nacional, acompañado de memoria en la que se alude a diferentes características del glaciarismo cuaternario.

\section{EL AREA DE ESTUDIO}

Se localiza en el Pirineo central español, en torno al macizo de Monte Perdido, y comprende integramente las cuencas de los ríos Arazas (o valle de Ordesa) y Bellos (o valle de Añisclo), la cuenca superior del río Cinca (o valle de Pineta) y buena parte de la cuenca del río Yaga (o valle de Escuaín). También se ha incorporado el valle del Ara que, aunque fuera de los límites del Parque Nacional, es imprescindible para disponer de una perspectiva global sobre los fenómenos glaciares. Este territorio pertenece a las llamadas Sierras Interiores Pirenaicas, alineación estrecha y alargada en sentido NNW-SSE, compuesta fundamentalmente por calizas, con importantes intercalaciones de areniscas y calizas margosas, de edad cretácica y eocena, formando en el macizo de Monte Perdido importantes apilamientos de pliegues cabalgantes hacia el Sur y superpuestos en sucesivos mantos de corrimiento. Esencialmente puede afirmarse que se trata de un macizo calcáreo, intensamente karstificado y hendido por profundos cañones en los que no siempre es fácil jerarquizar 
la importancia de lo fluvial, lo glaciar y lo meramente kárstico. Hacia el Sur las calizas se hunden por debajo del flysch, intensamente plegado pero dominado por un relieve de divisorias romas y pendientes mucho más suaves y regularizadas. Fragmentos aislados de flysch se localizan sobre las calizas, en Tobacor y Sierra Custodia.

A lo largo del macizo de Monte Perdido, tanto en su cara norte como en la sur, se suceden ininterrumpidamente los circos glaciares, alguno de grandes dimensiones, como el de Marboré, y otros más pequeños pero de formas muy bien definidas, como el de Soum de Ramond o los de la vertiente septentrional de las Tres Marías. Desde los circos descendían las masas de hielo en grandes cascadas de seracs hasta concentrarse en los valles, donde han dejado nítidas formas en artesa, no sin antes modelar algunas cubetas de sobreexcavación, probablemente de origen glaciokárstico, como las de Salarons, San Ferlús y Millaris.

\section{LA MAXIMA EXTENSION DE LOS GLACIARES}

En el mapa se ha reconstruído la extensión de los glaciares en el momento del máximo, a partir de la localización de los depósitos morrénicos más externos. Uno de los problemas más importantes para esta reconstrucción estriba en la ausencia de vallums terminales y por lo tanto los límites frontales deben ser supuestos muy aproximadamente, unas veces mediante restos demasiado dispersos y otras mediante la prolongación descendente de los cordones morrénicos laterales.

El valle del Ara, alimentado desde la vertiente oriental del macizo de Panticosa, Tendeñera, el Viñamala y, por medio del valle de Ordesa, desde el macizo de Monte Perdido, alcanzó notables dimensiones. El glaciar del Ara, con una potencia de más de $400 \mathrm{~m}$ inmediatamente aguas abajo de la incorporación de Ordesa, fue dejando potentes morrenas lateras que alcanzan su mejor expresión en la confluencia con valles afluentes (Diazas, Furco, Buesa y Sarvisé), aunque existen también empastes morrénicos más locales por toda la ladera, algunos de ellos derrubiados. En algún caso esas morrenas bloquearon la salida de las aguas de escorrentía, dando lugar a pequeños lagos que fueron rellenados de sedimentos, primero glaciolacustres y luego torrenciales. Así se observa, aunque con dificultad, en Diazas, donde la morrena lateral tiene una enorme envergadura y gran pendiente hacia el valle del Ara. En un pequeño corte se aprecia que el llano de Diazas culmina con una cumulación torrencial, heterométrica, bastante caótica y poco rodada, integrada por materiales procedentes del flysch, es decir, con origen en la propia cuenca del barranco de Diazas. Pero inmediatamente por debajo, apenas visible, aparece un depósito grisáceo con predominio de limos y arcillas, cuyo origen es innegablemente glaciolacustre. 


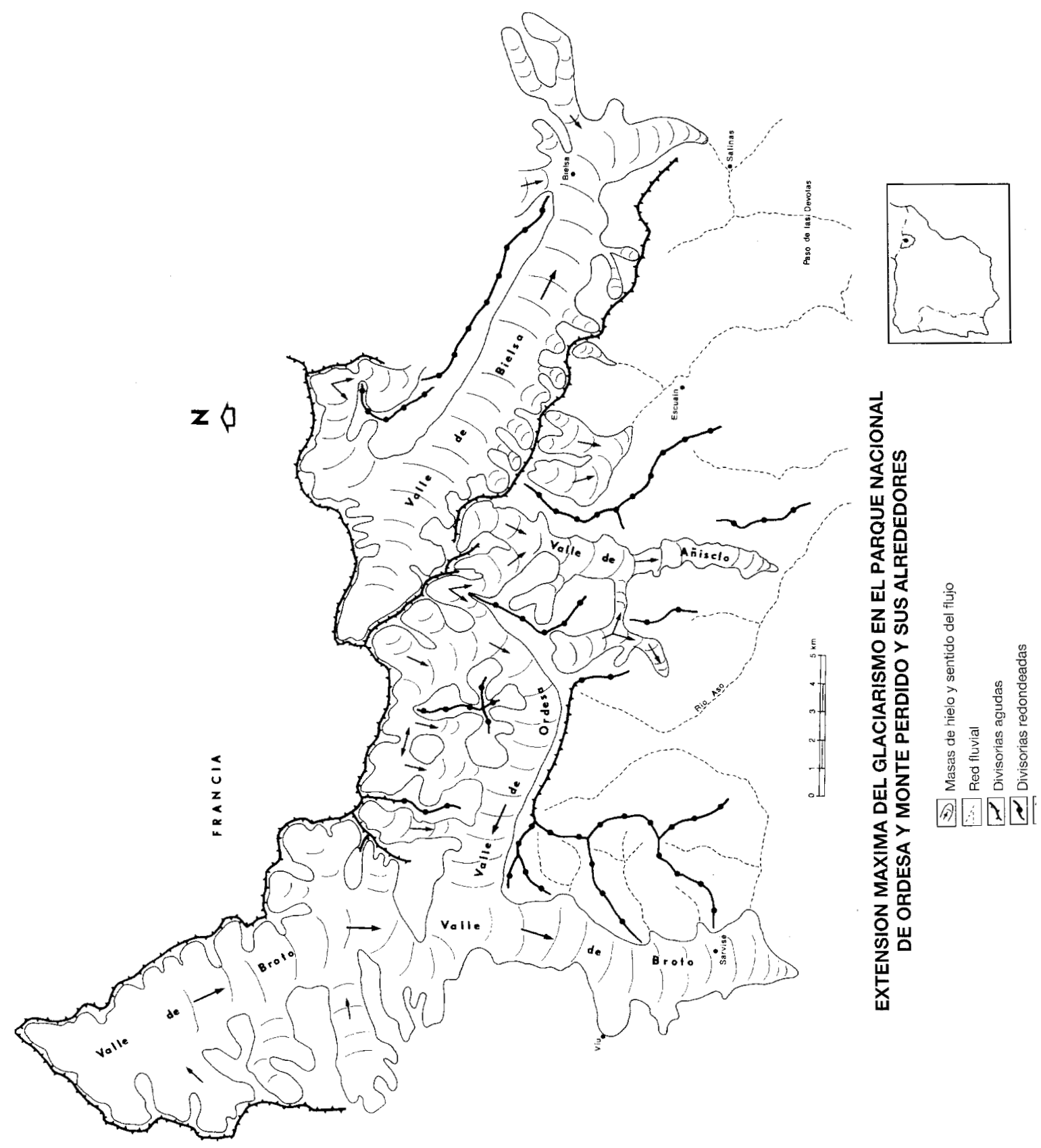


Aguas abajo de Diazas se identifica bien una serie de cordones morrénicos tanto en la margen derecha, donde bloquearon -por medio de la morrena de Viu- la salida del valle de Linás de Broto (ver Serrat et al., 1983), como en la izquierda. En la margen derecha dos cordones laterales muy claros (Viu y Frajén) permiten establecer al menos dos fases bien diferenciadas. En la izquierda se distinguen tres cordones en Furco y en Buesa, aunque no tienen gran continuidad. El más bajo -y, por lo tanto, el más reciente- desciende rápidamente en altitud, prologándose por el cordón de Sarvisé; la forma que dibuja y la baja altura que posee respecto al fondo del valle hacen suponer que en este sector tenía su terminación, coincidiendo con un ensanchamiento del glaciar. Los arcos superiores penetrarían algo más en el valle del Chate (de hecho hay bloques morrénicos sueltos en el tramo final de este valle y en su cono de deyección) y avanzarían hacia aguas abajo, hasta un punto no determinado, entre Broto y Fiscal, tal como surgirió Penck (1883), a una altitud de $850 \mathrm{~m}$. La existencia de laderas con formas similares a la de una artesa glaciar aguas abajo de Sarvisé hace sospechar que la acción glaciar no se limitó a un mero avance de corta duración.

En el valle de Ordesa no se han identificado depósitos morrénicos correspondientes a los momentos próximos al máximo. No puede haberlos frontales, pues el glaciar de Ordesa se incorporaba al del Ara, y los laterales -que formarían, de haberse depositado, modestas placas adosadas a las paredes del valle- no han podido conservarse fácilmente por las fuertes pendientes de las laderas. Es interesante señalar, no obstante, que durante el máximo el glaciar de Ordesa tenía una excelente alimentación desde la vertiente meriodinal del macizo de Monte Perdido, con una lengua principal procedente del circo del Cilindro y del circo del Lago Helado (entre El Cilindro y Monte Perdido), a la que se añadían las lenguas procedentes de los elevados relieves situados al Oeste del pico de Marboré, incluyendo El Casco y Taillón. Los hielos descendían hasta las cubetas glaciokársticas de San Ferlús, Millaris y Salarons y desde allí hasta el glaciar de Ordesa por Cotatuero -donde se unían las dos primeras lenguas- y Aguas Tuertas o Carriata, dando lugar a impresionantes valles colgados que engrosaron notablemente el espesor original del glaciar de Ordesa. También el pequeño macizo de Tobacor contó con sus correspondientes glaciares que descendian hacia la cabecera de Ordesa y Millaris, muy probablemente limitados en el tiempo al máximo glaciar.

En el valle de Añisclo es igualmente evidente la ausencia de depósitos morrénicos terminales, aunque puede deducirse con mucha aproximación el límite más externo alcanzado por los hielos. Un kilómetro aguas arriba del Puente de San Urbez se localizan pequeñas acumulaciones de innegable origen glaciar, parcialmente derrubiadas, a unos $900 \mathrm{~m}$ de altitud, muy cerca de lo que debió ser el extremo terminal del glaciar de Añisclo; en la misma pared se aprecian evidencias de pulimento por el hielo. Inmediatamente aguas abajo cesan las huellas de paso de un glaciar, al desaparecer la forma de artesa del valle -muy bien definida hacia aguas arriba- y 
precipitarse el río Bellos en una espectacular garganta fluvial. No hay ninguna evidencia de que el glaciar de Añisclo llegase hasta la confluencia con el barranco de Aso, donde no se ha encontrado ningun bloque o depósito de origen glaciar. En todo caso, el glaciar de Añisclo, bien alimentado en cabecera desde el circo de Soum de Ramond, desde las laderas orientales de este último pico y desde el circo de Añisclo propiamente dicho, careció hacia aguas abajo de otros tributarios importantes, salvo la modesta incorporación de hielo desde el barranco Pardina.

El valle de Escuaín soportó una glaciación bastante moderada, aunque conserva importantes depósitos y, localmente, muestra una morfología típica glaciar. Debe tenerse en cuenta que las únicas zonas posibles de acumulación de nieve y hielo están en la vertiente solana de las Tres Marías, en una exposición muy favorable a la ablación del hielo. Las lenguas emitidas eran cortas y seguramente poco potentes, una con origen en la Montaña de Sesa y la otra en Gurrundué. La zona terminal estaba situada poco más abajo de los actuales llanos de la Valle, donde existen varios arcos bastante bien definidos, uno de ellos, el principal, orientado de Norte a Sur y por lo tanto procedente de Gurrundué. Este arco bloqueó la salida del otro valle glaciar de Escuaín dando lugar muy probablemente a un pequeño lago ya colmatado. En este último valle existen varios cordones morrénicos bien conservados, con una colina alargada en la margen izquierda que se adosa a la base caliza de las Tres Marías y otras acumulaciones más voluminosas, aunque posteriores, al pie de la Montaña de Sesa, ya señaladas en el mapa de Barrére (1971).

Tampoco es fácil reconocer el límite más externo del glaciar de Pineta o del Cinca, ni su relación con el que descendía por el valle del Cinqueta, cuyas morrenas son descritas por Nussbaum (1949). Penck (1883) insinuó la posibilidad de que llegase hasta el Desfiladero de las Devotas, atendiendo a la existencia de bloques de ofita, a unos 100 metros por encima del fondo del valle. Nicolás Martínez no descarta el que este depósito pertenezca a una terraza antigua del Cinca. Es cierto que el Desfiladero de las Devotas parece encajarse a modo de garganta subglaciar en un valle con forma de artesa. Sin embargo, un reconocimiento detallado de los depósitos que tapizan las laderas y fondo de valle desde la salida de las Devotas hacia aguas arriba obliga a descartar el paso de un glaciar más abajo del cruce de Salinas, donde el río Cinqueta se incorpora al Cinca. En primer lugar, no existe entre Salinas y las Devotas ningún depósito morrénico ni se han encontrado restos de bloques permotriásicos o graníticos procedentes de la cabecera- en brechas periglaciares o en conos de deyección, donde hubieran podido depositarse por caida o arrastre torrencial desde una posible morrena lateral. En segundo lugar, unos 250 metros aguas abajo del cruce de Salinas existe a 790$800 \mathrm{~m}$, a 15-20 metros sobre el cauce actual, una terraza con numerosos cantos y bloques procedentes de la cabecera del valle (granito, areniscas del Permotrías ...), con centil superior a $20 \mathrm{~cm}$ y cemento arenoso muy abundante, que interpretamos como 
fluvioglaciar proximal, no muy alejada del frente glaciar. Sin embargo no hemos constatado la presencia de bloques en las laderas que dominan dicha terraza.

Poco menos de $1 \mathrm{Km}$ aguas abajo de la anterior aparecen otros depósitos de terraza (corte de San Marcial, ver Figura 1), entre 760 y 780 m. Al igual que en el caso anterior no se puede observar la base de la terraza.

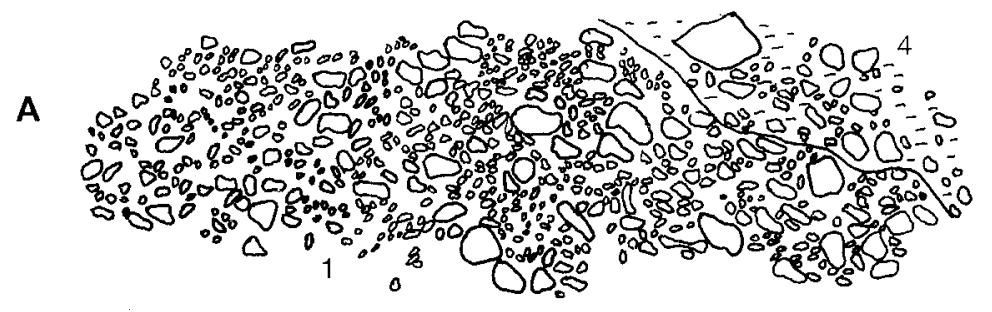

B

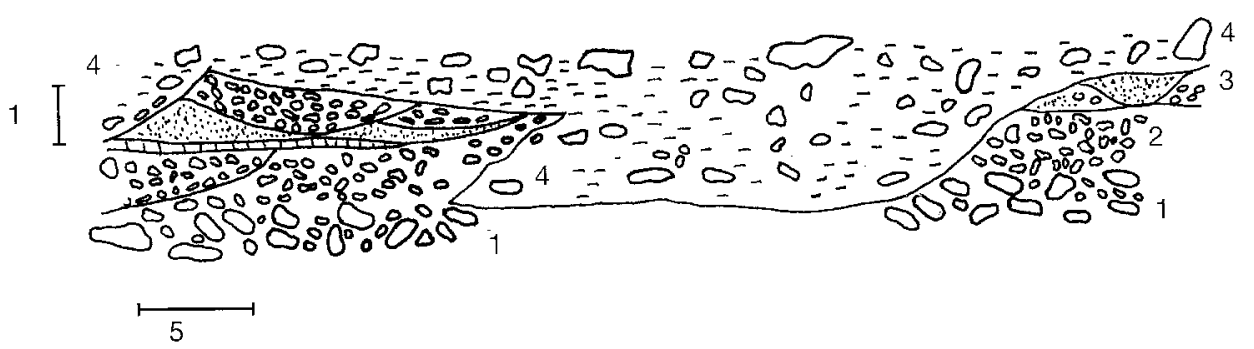

Figura 1.- Sección esquemática de la terraza fluvioglaciar del valle del Cinca en A) Hostal de Salinas y B) San Marcial: 1) Fluvioglaciar proximal. 2) Fluvioglaciar. 3) Depósitos fluviales en paleocanales. 4) Depósitos de ladera.

En los niveles visibles se aprecia, de abajo a arriba:

1) Cantos y bloques muy heterométricos, centil $>20 \mathrm{~cm}$, con cemento arenoso. Lo atribuimos a un fluvioglaciar proximal.

2) Nivel con cantos imbricados, centil mediano $(15 \mathrm{~cm})$, que atribuimos a un fluvioglaciar más alejado que el de la fase 1. 
3) Nivel de paleocanales, donde alternan gravas y arenas con estratificación cruzada y ripples. Se trata de una sedimentación fluvial en cauce anastomosado (braided). La influencia del glaciar se deja sentir menos que en las fases anteriores.

4) Brechas de ladera. Cantos y bloques de origen local, calizos y angulosos, cemento arcilloso rojizo abundante. Localmente aparecen cantos fluviales, lo cual, unido a que el nivel 2 se indenta en algunos lugares con el 4, implica la coetaneidad de la base de estos depositos de ladera con los niveles 2 y 3 de la terraza de $20 \mathrm{~m}$. La parte superior de este depósito de ladera es posterior a la terraza, a la que recubre.

En el desfiladero de las Devotas sólo apreciamos la existencia del nivel 1 y de un derrubio estratificado de ladera, donde alternan capas con matriz arcillosa con otras de estructura abierta, sólo ocasionalmente con cementación secundaria por carbonatos. Localmente aparecen grandes bloques de caliza, de orden métrico, caidos de los escarpes cercanos, que finalmente se hacen más abundantes, sustituyendo a los derrubios estratificados.

Estas diferencias con el modelo de cuatro niveles descrito en San Marcial se pueden atribuir a distintas dinámicas, tanto de vertientes como fluviales, en función de la estrechez mayor o menor del cauce, así como a cambios en la litología.

En la boca Sur del túnel de las Devotas se aprecia la presencia de cantos procedentes de la cabecera del valle a más de $20 \mathrm{~m}$ sobre el cauce actual del río, dentro de los derrubios de ladera. Puede atribuirse a que estos los englobaron a partir de la parte más alta de la terraza descrita, o procedentes de una terraza anterior (menos probable). No consideramos que sean herencia directa del paso del hielo por la zona ya que su índice de desgaste es alto y no presentan estrias. Unicamente facetas muy planas indican su paso por el hielo, lo cual también se da en terrazas fluvioglaciares.

De lo anterior cabe deducir que nos hallamos ante una terraza fluvioglaciar (o por lo menos la parte alta de ésta), que coexiste con unas brechas de clima frío, siendo finalmente recubierta por éstas. Parece claro que los glaciares del Cinca y del Cinqueta tuvieron su fase de estacionamiento cerca de la confluencia de ambos valles, a una altitud algo superior a los $800 \mathrm{~m}$. Si el hielo en una fase de expansión anterior al retroceso previo a la fase de estacionamiento llegó hasta las Devotas dejando bloques allí, como sugiere Penck, es cuestión aún no demostrada.

Por último, a pesar de sus modestas dimensiones, es interesante señalar la existencia de un glaciarismo independiente que se desarrolló en torno a Sierra Custodia, especialmente en la cabecera del barranco Pardina, con origen en el amplio 
relieve de Cuello Gordo, entre Custodia y Mondicieto. La posible penetración de hielo desde el valle de Ordesa, por medio de una difluencia, -aunque topográficamente no del todo imposible- debe descartarse pues en los depósitos abandonados por este glaciar no se han encontrado areniscas del Maestrichtiense (o de Marboré) ni calizas blancas del Paleoceno de la Formación Salarons, típicas de la cabecera de Ordesa. Desde Cuello Gordo el hielo se desplazaba hacia el llano de Cuello Arenas, donde se le añadía un pequeño glaciar procedente de la vertiente sudoriental del Mondicieto (Llano Tripal). Desde allí, donde hay varios depósitos morrénicos correspondientes a una fase de estancamiento o reavance tardío, la lengua de hielo se subdividía en dos ramas, una que descendía por Pardina hacia Añisclo y otra por Cuello Arenas hacia el valle de Aso o valle de Vió. En este último puede identificarse una gran morrena atravesada y destruida en su parte frontal por el barranco de Caldaruelo -que alcanza su extremo terminal a $1.650 \mathrm{~m}$, y otros arcos internos próximos, todo ello en una exposición muy abierta y soleada, propensa a la ablación rápida.

\section{OTROS DEPOSITOS MORRENICOS}

Todos los valles poseen huellas de varias fases de estabilización y retroceso posterior, aunque las acumulaciones frontales sólo existen en las zonas más elevadas, a veces dentro de los mismos circos. No obstante, algunos de los depósitos citados en la bibliografía corresponden a morrenas laterales derrubidas o transportadas por torrentes. Ese es el caso del cono de deyección de Torla, citado como una morrena frontal del valle de Ordesa (Hernández Pacheco, 1920) por la abundante presencia de bloques morrénicos arrastrados desde la ladera. Este mismo autor cita otra morrena dentro del valle de Ordesa, junto a la cascada del Abanico, "algo más abajo de la cascada del Estrecho, a los 1.420 metros de altitud" (p.275), que en nuestra opinión se trata de un depósito caído desde la ladera, manteniendo todavía muy poca selección granulométrica. Igualmente, Hernández Pacheco y Vidal Box (1946) señalan que el depósito situado en Bielsa (valle del Cinca), inmediatamente por encima del pueblo, correspondería a una morrena frontal, por la presencia de grandes bloques empastados en material más fino. Este depósito es en realidad un gran deslizamiento que afecta a la morrena lateral, caída hasta el fondo del valle en forma de lóbulo, como lo es también otro gran paquete morrénico, aguas arriba del anterior, en la margen derecha de la presa de Pineta. Un cono de deyección activo se forma precisamente junto a este embalse por erosión torrencial de la morrena lateral derecha de Pineta. Tales deslizamientos ocuparon todo el fondo del valle -como lo prueba el corte que efectúa el río Cinca-, y bloquearon la circulación normal de este último, dando lugar a un represamiento que favoreció localmente la sedimentación aguas arriba. 
En la margen izquierda, inmediatamente aguas abajo de la presa, existe una colina rocosa rematada por un depósito morrénico. Por sus características podría tratarse de un pequeño fragmento de arco frontal, pero es más probable su caída desde los escarpes situados en la ladera izquierda.

En cambio, el valle de Pineta, bajo el circo del mismo nombre, mantiene muy bien las morrenas frontales de una fase muy tardía (la llamada fase de glaciares de altitud), muy probablemente contemporáneas de la morrena de la confluencia de Mulleres y Conangles, en el alto Noguera Ribagorzana (Vilaplana, 1983), de las morrenas fronto-laterales del valle de Fontfreda (Bordonau, 1987) y de las morrenas de Izas, Piedrafita y Acherito, en los valles del Aragón, Gállego y Subordán. Al periodo Tardiglaciar se atribuye por su aspecto la morrena gris del circo de Marboré (Nicolás-Martínez, 1981), alimentada con bloques de calizas eocenas caídas desde los escarpes más elevados de Monte Perdido y del Cilindro, configurando, al menos parcialmente, un glaciar cubierto. Por su posición, estado de conservación y aspecto general, las morrenas de la cara sur del Pico Bacillac -formadas por una corta lengua llegada desde el Taillón- serían contemporáneas de la anterior. En cambio, no hay evidencias de una fase de glaciares rocosos, que requieren la presencia de rocas muy tectonizadas capaces de producir instantáneamente enormes volúmenes de bloques en forma de coladas de piedras.

De difícil determinación cronológica son los restos dispersos en las zonas altas de Ordesa, entre Góriz y el llano de Salarons. Todos pertenecen a fases muy tardías, probablemente sin especial significación paleoclimática. El valle afluente de Salarons es el que conserva mayor número de depósitos, correspondientes a un momento en que este glaciar estaba desconectado del principal de Ordesa. El llano de Millaris tiene también pequeños depósitos, y a altitud similar hay nuevos restos cerca del refuguio de Góriz y en los rellanos situados entre este último y la Plana de San Ferlús.

Más recientemente, la Pequeña Edad del Hielo ha dejado abundantes restos morrénicos en todo el macizo de Monte Perdido, desde el Taillón al Soum de Ramond. Los mejores ejemplos están en el circo de Marboré, con un prolongado cordón que marca el máximo de ese período, en un momento en que los glaciares del Cilindro y de Monte Perdido formarían una masa de hielo única, y varios cordones internos. En la cara meridional hay algunos ejemplos de depósitos recientes muy definidos, como es el caso de la morrena situada a la salida del circo de Soum de Ramond, o los de las morrenas del circo meridional de Marboré-Cilindro, así como la del Taillón. 


\section{CONCLUSIONES}

1. La altitud alcanzada por las lenguas glaciares principales del Parque Nacional de Ordesa y Monte Perdido en el momento del máximo coincide en todos los valles en torno a 850-950 metros (Ara, Añisclo y Cinca), siendo el valle del Ara el que descendió hasta niveles más bajos. En el valle de Escuaín el glaciar se detuvo hacia 1.300-1.400 m, mientras que el glaciar de Pardina-Vió llegó sólo hasta $1650 \mathrm{~m}$, en razón de la insolación y menor altitud de su cabecera. En todos los valles, excepto en el de Pardina-Vió, faltan las morrenas frontales del momento del máximo, por lo que la zona terminal sólo puede deducirse a partir de la posición de depósitos laterales o por la presencia de bloques morrénicos en brechas periglaciares o en conos de deyección.

2. En la fase del máximo pueden identificarse varios estadios, en razón del número de morrenas laterales perfectamente individualizadas. Así, en el valle del Ara se distinguen dos cordones muy claros en la margen derecha -en la confluencia con el valle de Linás- $y$ tres en la margen izquierda, aguas abajo de Broto.

3. La fase de glaciares de altitud, entre 1600-1700 m, está representada por la morrena al pie del circo de Pineta y probablemente por pequeños restos aguas abajo de Fuen Blanca en el valle de Añisclo.

4. La fase de morrenas de circo -quizás correspondiente al Tardiglaciar-, con importantes acumulaciones heterométricas muy informes, se halla representada por la morrena gris del circo de Marboré y otros restos de la vertiente solana del macizo de Monte Perdido, especialmente el gran depósito entre El Taillón y El Casco, al pie del pico Bacillac.

5. Por último, la Pequeña Edad del Hielo ha dejado numerosos restos con formas arqueadas muy netas, sobre todo en el circo de Marboré, en el de la cara sur del Cilindro-Marboré y en el de Monte Perdido-Soum de Ramond. Tales morrenas estaban en contacto con los glaciares a mediados del siglo pasado.

\section{BIBLIOGRAFIA}

BARRERE, P. (1971): "Le relief des Pyrénées Centrales franco-espagnoles", Institut de Géographie, Université de Bordeaux.

BORDONAU, J. (1987): "El glaciarismo cuaternario en la cuenca del río Nere (Vall d'Arán, Pirineos centrales)", Actas de la VII Reunión del Cuaternario Ibérico, AEQUA, 163-166, Santander. 
BOYE, M. (1952): "Névés et érosion glaciare", Revue de Géomorphologie Dynamique, 20-36.

GARCIA RUIZ, J.M. y MARTI BONO, C. (1993): Mapa Geomorfológico del Parque Nacional de Ordesa y Monte Perdido", Estudio para ICONA, Instituto Pirenaico de Ecología, 118 p. más un mapa a escala 1:25.000, Zaragoza.

GOMEZ DE LLARENA, J. (1936): "Algunos datos sobre el glaciar actual del Monte Perdido (Pirineos)",Boletin de la Real Sociedad Española de Historia Natural, 36, 327-343.

HERNANDEZ PACHECO, E. y VIDAL BOX, C. (1946): "La tectónica y la morfología del macizo del Monte Perdido y de las zonas de cumbres inmediatas en el Pirineo central", Pirineos, 4, 69-108.

HERNANDEZ PACHECO, E. (1920): "Comunicación respecto a los parques nacionales y a los monumentos naturales de España", Boletín de la Real Sociedad Española de Historia Natural, 20, 267-282.

NICOLAS MARTINEZ, P. (1981) "Morfología del circo de Tucarroya (Macizo de Monte Perdido, Pirineo aragonés)", Cuadernos de Investigación Geográfica, 7, 51-80.

NUSSBAUM, F. (1949): "Sur les traces des glaciers quaternaires dans la region de l'Aragón", Pirineos, 13-14, 497-518.

OBERMAIER, F. (1921): "El glaciarismo cuaternario en el valle del río Ara y en el Parque Nacional de Ordesa (Pirineos)", Bol. Soc. Ibérica de Ciencias Naturales, 20, 106-110.

PENCK, A. (1883): "Die Einzeit in den Pyrenaen", Mitt. Ver. Erdk., Leipzig. "La période glaciaire dans les Pyrénées", Traducido al francés en el Bulletin de la Societé d'Histoire Naturelle de Toulouse, 19, 105-200.

SERRAT, D.; VILLAPLANA, J.M. \& MARTI, C.E. (1983): "Some depositional models in glaciolacustrime environments (Southern Pyrenees)", En, EVENSON, E.B.; SCHLUCHTER, CH. \& RABASSA, J. (Eds.): Tills and related deposits, 231-244, Balkema, Rotterdam.

VILAPLANA, J.M. (1983): "Estudi del glaciarisme quaternari de les altes valls de la Ribagorza", Tesis Doctoral, Facultat de Geología, Universitat de Barcelona, 322 p., Barcelona. 